\title{
Traduire
}

Revue française de la traduction

$218 \mid 2008$

De traduction en retraduction

\section{Traduire et retraduire les textes de l'Antiquité gréco-latine}

Anne-Marie Ozanam

\section{(2) OpenEdition}

Journals

Édition électronique

URL : http://journals.openedition.org/traduire/893

DOI : 10.4000/traduire.893

ISSN : 2272-9992

\section{Éditeur}

Société française des traducteurs

\section{Édition imprimée}

Date de publication : 15 juin 2008

Pagination : 14-28

ISSN : 0395-773X

\section{Référence électronique}

Anne-Marie Ozanam, "Traduire et retraduire les textes de l'Antiquité gréco-latine », Traduire [En ligne], 218 | 2008, mis en ligne le 15 juin 2008, consulté le 03 mai 2019. URL : http:// journals.openedition.org/traduire/893; DOI : 10.4000/traduire.893 


\title{
Traduire et retraduire les textes de l'Antiquité gréco-latine
}

\author{
AnNe-Marie Ozanam \\ À la mémoire de Pierre Vidal-Naquet, \\ avec gratitude
}

La tâche du traducteur de grec ancien ou de latin ne semble pas, à première vue, obéir à une nécessité pressante. Tout n'est-il pas déjà traduit ? Et dans ce cas, quel est l'intérêt de revenir sur des œuvres que l'on connaît depuis des siècles? Pour paraphraser La Bruyère, tout est fait, et l'on vient trop tard... S'il s'agit seulement de dépoussiérer les versions précédentes en remettant au goût du jour les expressions qui ont vieilli, en remplaçant, par exemple, le terme de "piéton ", qui ferait maintenant faux-sens, par celui de "fantassin", ou "se faire bien venir " par " se faire bien voir ", le travail ne dépasse guère ce que l'on appelle actuellement le rewriting.

C'est sans doute ce que pensent la plupart des éditeurs qui montrent le peu d'estime en lequel ils tiennent l'antiquisant par le traitement financier qu'ils lui accordent. Alors que le traducteur de langues vivantes est payé au feuillet, et que le prix de ce feuillet augmente si la langue est dite " rare ", hellénistes et latinistes sont en général rémunérés - s'ils le sont ! - au forfait, et leur traduction "vaut " beaucoup moins par elle-même que par les outils qui l'accompagnent : introduction, commentaires, notes, index, bibliographie...

Du reste, l'antiquisant semble partager cette opinion : il se définit rarement comme traducteur, et participe encore plus rarement - par modestie ou par mépris ? - aux associations et aux rencontres de ses pairs spécialistes de langues modernes. Comme s'il ne faisait pas le même travail qu'eux. Comme si son activité de traduction n'était qu'une partie, bien secondaire, de ses travaux. 
Or, contrairement à ce que l'on croit, les textes grecs et latins sont encore loin d'être tous traduits. L'immense naufrage qui a englouti tant d'écrits continue à rejeter, de temps en temps, quelques épaves : la redécouverte la plus éclatante a été, dans les années 1950 , celle du texte entier du Dyscolos de Ménandre, qu'on croyait avoir à jamais perdu, et qui a pu être publié en 1959 ...

Et surtout, pendant des siècles le corpus est resté obstinément réduit, particulièrement en France, à de sacro-saints classiques, loin desquels il semblait impie, ou du moins peu sérieux, de s'aventurer. Il suffit de regarder les programmes officiels et les anthologies scolaires pour constater que ce sont presque toujours les mêmes auteurs qui sont sollicités(1) et qui ont très vite constitué une sorte de "canon " profane. En grec, les orateurs attiques, Platon, Xénophon, Sophocle, Euripide... En latin, Cicéron, César, Tite-Live, Virgile, Horace, Suétone, Tacite... Il est intéressant en ce sens de consulter le catalogue des "petits classiques " Hachette (établi en 1904, mais la collection a commencé vers 1880), et de comparer le Programme des Lycées et Collèges du 7 mai 1931 avec l'Arrêté du 8 juin 1966. On s'aperçoit que le choix de textes n'offre guère de différence significative. La littérature dite tardive est presque totalement absente. Quant aux écrivains du Moyen Âge et de la Renaissance - ces derniers ont un style pourtant si pur, si proche des classiques! -, on n'en trouve pas trace. Dans la préface de février 1934 de son dictionnaire, qui sert encore de référence, notamment pour tous les concours, Félix Gaffiot n'évoque-t-il pas « la latinité au sens vrai du terme, c'est-à-dire celle qui s'étend de Plaute à Tacite».

Dans le même esprit, les outils dont disposent les étudiants sont bien désuets. À l'époque d'Internet(2), le grand événement éditorial de ces dix dernières années a été, en France(3), la réédition, presque à

(1) Voir Chervel : 1986 ; Carré : 1887.

(2) Signalons cependant la parution récente, en 2007, du Dicolatin, dictionnaire français-latin en ligne (www.dicolatin.com).

(3) À la différence notamment de l'Angleterre où les éditions d'Oxford University Press ont publié en 1982 un dictionnaire latin-anglais très moderne, rédigé par une nombreuse équipe. 
l'identique, de deux dictionnaires, dus chacun à un seul homme - donc malgré l'érudition de celui-ci, forcément limités : "le " Gaffiot, que nous venons d'évoquer, et " le " Bailly. Or ces dictionnaires s'adressaient explicitement à de jeunes élèves. Dans la préface de son ouvrage (qui date de 1894), Anatole Bailly précise qu’il a supprimé les mots qui " ne sont d'aucune utilité pour les élèves de lycées et de collèges ".

Or ces manuels ne sont qu'un reflet du conservatisme qui étouffe trop souvent le petit monde des antiquisants français. En dépit des travaux des chercheurs, la majorité des enseignants, des étudiants et du public cultivé continue à ignorer la période hellénistique, le «Bas »-Empire, le monde byzantin et les prétendues ténèbres médiévales. Certains s'attachent à combler ces lacunes, mais il y a fort à faire, et nombreux sont les écrits qui restent à traduire. Comme le suggère le titre d'une collection des Belles Lettres, la " Roue à livres(4) " doit encore tourner pour que sortent enfin de l'oubli des textes injustement méconnus.

Tous n'appartiennent d'ailleurs pas aux époques dites obscures. Même le " siècle de Périclès ", que l'on croit si bien connaître, s'est longtemps résumé à l'image lumineuse que suggère la Prière sur l'Acropole de Renan : celle d'une cité raffinée, cultivée, vouée à la " déesse dont le culte signifie raison et sagesse ", une cité libre et fière, fortement idéalisée, où philosophes et orateurs dissertent gravement en déambulant entre des colonnes de marbre blanc... Or ces colonnes étaient peintes, en des couleurs qui peut-être froisseraient le goût que nous croyons avoir hérité des Antiques. Et Athènes avait sa face irrationnelle, sa face d'ombre, qu'on a trop occultée : sa cruauté, ses superstitions, ses sectes étranges, ses totems et ses tabous, ses mauvais lieux " hors les murs", peuplés de sorcières, de prostituées, de nécromants et d'aventuriers, moins décoratifs sans doute, mais plus vrais que les bergers d'Arcadie(5). Il en va de même pour la Rome républicaine ou impé-

(4) Nous y avons nous-même publié la première traduction française complète d'Alciphron (Ozanam : 1999).

(5) Nous avons tenté, à la suite de Bernand : 1991, et de Ginzburg :1989, d'en donner une idée (Charvet et Ozanam : 1994). 
riale, beaucoup plus violente et sauvage qu'on ne le dit. Il est donc très important, même pour la compréhension des grandes œuvres classiques, lesquelles ne sont pas forcément si étrangères qu'on l'imagine à ces activités marginales(6), que des traducteurs portent à la connaissance du public les documents qui en témoignent.

Quant aux textes qui nous sont connus depuis des siècles, les retraduire périodiquement est une nécessité. Et il s'agit de beaucoup plus que de moderniser des traductions dont seule la langue aurait vieilli. Nous avons éprouvé personnellement qu'il est incomparablement plus difficile de travailler sur une œuvre qui a déjà été - et parfois à de nombreuses reprises - traduite que de la déchiffrer pour la première fois.

La véritable retraduction (qui s'oppose en tous points au rewriting) implique en effet une remise en question fondamentale de ce qui nous a été transmis comme une évidence et un dogme. Elle nous impose de nous libérer d'idées toutes faites et de terminologies que nous avons héritées, sans nous en apercevoir, des traductions précédentes, et plus largement, de toute une tradition scolaire : chargées de leur propre idéologie et de celle de leur époque, elles font écran entre nous et le texte que nous devons faire entendre.

C'est surtout avec les Vies parallèles de Plutarque que nous avons pris conscience de ces difficultés. L'ouvrage a en effet été très vite considéré par les Modernes comme une somme privilégiée de connaissances sur le monde grec et latin. C'est pourquoi, très vite aussi, la traduction de certains termes, notamment tous ceux qui ont trait aux institutions, s'est transmise à travers les siècles, de traducteurs en lecteurs, avec une autorité particulièrement contraignante.

La première traduction, sans doute la plus célèbre, celle de Jacques Amyot, date de 1559. Plus de deux siècles auparavant, en traduisant

(6) Voir notamment Dodds : 1951 ; Vidal-Naquet : 1981 ; Lloyd : 1990. 
Tite-Live, Pierre Bercheure avait choisi de "naturaliser", sans les transposer, beaucoup de mots romains, pour en conserver le caractère étranger : auspices, augure, cirque, faction... Amyot adopte le parti inverse. Afin de rendre compréhensibles les coutumes de l'Antiquité gréco-latine à un public du XVIe siècle, il les rapproche le plus possible des réalités familières à ses contemporains. Ainsi fait-il de l'hipparchos le "capitaine général de la gendarmerie ", de la chlamyde un "manteau ducal", tandis que Romulus se trouve entouré de "gentilshommes". Comme l'écrit Pascal Payen, il "recrée Plutarque en l'enlevant à son temps $(7)$ ».

Du coup, dans un mouvement symétrique et complémentaire, les lecteurs des XVIe-XVIIe siècles s'approprient le Plutarque d'Amyot et celui des traducteurs suivants qui œuvrent dans le même sens(8). Ils s'identifient à ses héros qu'ils érigent en modèles. Brantôme puise dans les Vies parallèles la matière de sa Vie des hommes illustres. Le Grand Condé les emporte dans ses bagages. Shakespeare et Corneille leur empruntent la matière de plusieurs pièces... Au XVIIIe siècle encore, la projection affective reste très forte. Rousseau lit avec son père la traduction d'Amyot:il en retire un fervent enthousiasme pour l'Antiquité. "Que ne suis-je né Romain!" s'écrie-t-il. Et les Vies restent le modèle auquel se réferent les révolutionnaires, soucieux d'imiter les héros de Plutarque, et de faire de "belles morts ". Belles comme l'antique...

Or, si stimulantes qu'elles aient pu être pour l'imaginaire et pour l'affectivité, ces assimilations, qui provenaient d'anachronismes voulus par le premier traducteur afin de faciliter la lecture, ont fini par fabriquer une Antiquité à l'image des rêves des Modernes : un beau miroir conforme à leurs convenances, à leur morale, à leur esthétique, qui n’a plus grand-chose à voir avec la réalité historique.

(7) Payen : 2001.

(8) Voir notamment la traduction du pasteur Simon Goulart, 1585, évoquée par Payen : 2001. 
Un exemple, apparemment anecdotique, concerne le mot grec aulos. Cet instrument à anche double avait un mordant aigre et produisait un son strident $(9)$. Traduire aulos par "flûte", comme on le fait communément, semble bien inoffensif. Et pourtant, ce rapprochement a suggéré à des générations de lecteurs une image édulcorée de la musique antique, faite d'harmonies et de grâces un peu mièvres, gommant tout ce qu'elle pouvait avoir d'oriental et de violent.

C'est surtout dans le domaine des institutions que les traductions françaises ont longtemps suivi la route frayée par Amyot. Ainsi l'éphèbe est-il resté pendant des générations un "jeune homme", voire un "beau jeune homme", et il a fallu attendre la deuxième moitié du XXe siècle pour que l'on découvre la spécificité de cette institution athénienne qu'était l'éphébie : l'inscription de l'éphèbe se faisait à la majorité légale (18 ans) et l'éphèbe devait accomplir deux années de service(10). On pourrait multiplier les exemples : le stratège athénien était vu comme un simple "général ", alors qu'il s'agit d'abord d'un magistrat ; l'hétairie comme une " camaraderie " alors que la solidarité qu'elle implique ne repose pas seulement sur l'amitié ; l'agora ou le forum comme des "places publiques", sans tenir compte de leur rôle social, commercial et politique.

On le voit, à force de ramener l'autre au même, l'étranger au familier, l'inconnu au connu, traducteurs et lecteurs de Plutarque ont fini par créer un objet imaginaire, assez semblable à ce que les péplums ont longtemps proposé aux spectateurs : des toges ou des tuniques, des bijoux anciens, des lampes à huile, des colonnes, des gradins, quelques invocations à Zeus ou à Jupiter, mais pour le reste, un monde qui ressemble en tous points au nôtre.

(9) Voir Schaeffner : 1936, et Bélis : 1999.

(10) Voir Aristote, Constitution d'Athènes, 41 et Vidal-Naquet : 1981. 
Le déni de l'étrangeté du monde gréco-romain a été parfois si fort qu'il y a même eu des hellénistes pour refuser obstinément de se rendre en Grèce, de peur d'être "déçus "... Ils préféraient s'en tenir à leur rêve personnel, imaginer un pays poétique que les réalités matérielles ne risqueraient pas de gâter. Or une telle démarche, apparemment bien innocente, peut se teinter d'arrière-pensées plus inquiétantes...

La tentation a en effet toujours été grande de s'annexer l'Antiquité afin d'y retrouver non seulement des modèles, mais aussi d'imaginaires " racines" - et l'on sait à quel point ce mot peut avoir des connotations dangereuses...

La réception de la Germania de Tacite en fournit un exemple particulièrement éclairant. Cet opuscule n'était peut-être à l'origine qu'une conférence mondaine : Alain Michel imagine que Tacite a «loué une salle... envoyé des invitations et, vêtu d'une toge d'apparat, a lu son œuvre de sa plus belle voix devant l'élite mondaine de la cité(11) ». Ce texte propose aux Romains du Ier siècle un voyage exotique dans un monde " barbare ", inquiétant, aux frontières de l'Empire. Si sa valeur documentaire est indéniable(12), celle-ci est subordonnée à la leçon que le moraliste entend délivrer. Conformément à une tradition ethnographique qui remonte à Hérodote(13), les Barbares sont présentés comme des êtres purs, sains, proches de la nature, exempts des vices de la "décadence " : chez eux, pas d'adultère, pas de goût immodéré du luxe, une vie rude et vertueuse... Cette peinture de "bons sauvages" n'a sans doute que de lointains rapports avec ce qu'étaient vraiment les Germains : elle vise avant tout à l'édification morale.

Or, si peu réaliste qu'elle puisse être, elle a joué un rôle exceptionnel dans la formation des mentalités modernes. Sa redécouverte au milieu du XVe siècle (14), au moment où le Saint Empire romain germanique

(11) Michel : 1966.

(12) Voir Perret : 1949 ; Dumézil : 1959. Cependant, Grimal : 1990 voit dans l'ouvrage " une semi-fiction où... les ignorances sont voilées, où les connaissances certaines sont généralisées de manière abusive. "

(13) Voir Hartog : 1991.

(14) La première édition paraît à Bologne en 1472, mais le texte semble connu depuis 1458 (voir notre préface dans Ozanam : 2002). 
est en train de se disloquer, fournit aux Allemands un idéal qu'ils peuvent opposer à l'héritage de Rome dont se prévalent les Italiens. Dans la deuxième moitié du XVIe siècle les Protestants français y puisent eux aussi une image de leur résistance à Rome (15). Aux siècles suivants, les différents peuples européens, qui cherchent à se forger une identité nationale, cherchent un passé mythique auquel la rattacher : ils font souvent appel aux Germains de Tacite. Les Français leur relient étroitement les Francs dont ils se disent descendants. Ainsi le «temple des Druides " du parc d'Ermenonville, rendant hommage à " nos aïeux... ces braves Gaulois", reprend presque textuellement Germania(16). L'éloge que fait Rousseau dans l'Émile de l'allaitement maternel provient de la même source. Quant à Montesquieu, il n’avait pas classé l'ouvrage sous la rubrique Romanorum rerum scriptores, comme les autres ouvres de Tacite, mais sous l'intitulé Gallicarum rerum scriptores; il y voit le "fil d'Ariane " qui permet de comprendre les institutions des Francs et même le régime parlementaire de l'Angleterre : « Si l'on veut lire l'admirable ouvrage de Tacite sur les mœurs des Germains, on verra que c'est d'eux que les Anglais ont tiré l'idée de leur gouvernement politique. Ce beau système a été trouvé dans les bois(17).»

C'est avec le nationalisme allemand que la Germania trouve ses lecteurs les plus fervents. L'ouvrage de Tacite joue le rôle de «nouvel Évangile " : c'est "plus que de l'histoire, c'est de l'histoire sacrée $(18)$ ». Mommsen lui-même s'écrie : « Toujours, nous autres Allemands, nous nous trouverons unis dans la joie et la fierté de constater qu'un des meilleurs Romains, lorsqu'il vit décliner le soleil de sa nation, a ébauché de la nôtre un tableau qui a su réunir ses forces, des millénaires plus tard, dans le nouvel Empire allemand, mieux qu'au temps du Saint Empire romain germanique, en une grande œuvre: notre nation, dont l'avenir déterminera longtemps encore le destin du monde, plus même qu'elle ne l'a fait deux mille ans durant(19). "

(15) Voir Dubois : 1972.

(16) Voir Volpilhac-Auger : 1993.

(17) Esprit des Lois, XI, 6.

(18) Ridé : 1980.

(19) Discours prononcé le 21 janvier 1886 à l'Académie de Prusse. 
Cette lecture affective, fondée sur l'identification, devait conduire aux égarements que l'on sait(20)... Dépassant de loin les intentions de Tacite et les réalités germaniques du Ier siècle, traducteurs, commentateurs et lecteurs ont donc fini par fabriquer une "germanité " de pure fiction. On voit, par ce cas extrême, combien il peut être dangereux de solliciter l'Antiquité comme un modèle, de chercher en elle un miroir, voire une légitimité, bref de se l'annexer.

À l'inverse d'une telle attitude, et ce dès la fin du XVIIIe siècle, des traducteurs ont souhaité lui rendre sa voix propre et la découvrir véritablement, dans sa spécificité. Ainsi Goethe(21) écrit-il au Chancelier von Müller : "Dans la traduction, on ne doit pas s'engager dans une lutte immédiate avec la langue étrangère. On doit parvenir jusqu’à l'intraduisible et respecter celui-ci, car c'est là que résident la valeur et le caractère de chaque langue(22). " Un regard nouveau est posé sur le monde gréco-latin. Au lieu de le rapprocher du nôtre, on s'efforce au contraire de dégager ce qui l'en sépare, ce qu'il a de différent, voire d'incompréhensible(23).

Le danger de l'entreprise, bien sûr, est de sacrifier l'intelligibilité. La fidélité scrupuleuse à la lettre, voire au mot à mot, peut déboucher sur un charabia pseudo-mallarméen, ce qui serait une forme de trahison aussi nuisible que les ornements des belles infidèles, surtout pour un auteur aussi soucieux d'être clair et convaincant que Plutarque(24). Il faut donc raison garder. Le plus important, à notre sens, est de conserver l'ordre d'apparition des idées, souvent malmené dans les traductions anciennes. Surtout pas, bien sûr, l'ordre des mots - les systèmes

(20) Voir notamment Canfora : 1979.

(21) Voir ses analyses dans Le Divan occidental-oriental.

(22) Cité par Berman : 1984.

(23) Sur ce mouvement, voir notamment Berman : 1984 ; Meschonnic : 1999 ; les travaux de George Steiner...

(24) Il fait l'éloge de la clarté dans Sur les oracles de la Pythie, 406, e. 
de langue n'étant pas identiques -, mais les unités de sens : un élément qui était au début ne doit pas se retrouver après celui qui le suit. Il faut, dans la mesure du possible, respecter la sémantique des positions.

Quant au souci de préserver systématiquement les termes originels, il risque de conduire à une traduction qui restera illisible si elle n'est accompagnée d'un imposant appareil de notes. En ce qui concerne Plutarque, nous avons tenté de trouver un équilibre souvent délicat, en évitant à la fois l'obscurité et les assimilations abusives. Nous avons veillé, grâce aux conseils de Pierre Vidal-Naquet et de Claude Mossé, si attentifs à ces questions, à maintenir avec rigueur les termes institutionnels, à ne pas parler de "soldats " mais d' « hoplites", de " jeux ", mais de " concours" olympiques, de " courtisanes », mais d'" hétaïres ", de " mignons " mais $d^{\prime}$ " éromènes "...

Un problème assez piquant se posait cependant. Plutarque lui-même (et c'est l'idée fondatrice des Parallèles), quand il passe du monde latin au monde grec, pratique précisément cette technique du rapprochement, à laquelle notre équipe et nous-même tentions de résister. Ainsi le consul romain devient chez lui un archonte, le préteur un stratège, le tribun militaire un chiliarque ; il traduit le Sénat par la Boulé, le forum par l'agora, l'Afrique par la Libye, Jupiter par Zeus...

Que devions-nous faire ? Notre désir était grand de préserver ces assimilations, qui donnent aux Parallèles une saveur particulière, un peu exotique, en mêlant les deux cultures. D'autant que c'est un choix délibéré de notre auteur qui, lorsqu'il le souhaite, sait tout à fait employer les termes romains précis(25).

Pour finir, nous nous sommes rendue aux arguments de l'équipe : le public risquerait d'être déconcerté par "Zeus Capitolin », le "Champ d'Arès » et les " deux archontes » de Rome... Nous avons donc rejoint sur ce point la tradition, tout en ayant conscience (et en regrettant) de perdre ainsi le "grain" du texte. Du reste, dans leur traduction des 
Questions romaines, questions grecques, Michèle Nouilhan, Jean-Marie Pailler et Pascal Payen ont choisi de garder les termes grecs(26).

Une autre difficulté, plus profonde, tenait à certains termes qui mêlent politique et morale et qui peuvent être chargés d'une idéologie bien différente, selon la traduction qu'on adopte. Ainsi en est-il du mot démos, si important dans la langue grecque. Plutarque l'emploie le plus souvent de manière positive, au sens du populus latin, et il lui donne ainsi une légitimité forte. Mais c'est parfois la plebs, les gens de basse extraction opposés aux aristocrates. Enfin, il peut s'agir du vulgus ou de la multitudo, la populace aux instincts redoutables, prompte à se déchaîner, si facile à flatter et à manipuler...

Dans le même esprit, comment traduire démagogos? Le problème se pose avec particulièrement d'acuité dans la Vie de Périclès où le mot revient très souvent. S'agit-il, au bon sens du terme, de celui qui " sait conduire le peuple" ? Ou, au sens politique, du " chef du parti populaire " ? Ou enfin, selon l'acception moderne et péjorative, déjà largement attestée à l'époque, du "démagogue" ? Quant aux aristoi ce pluriel désigne-t-il les aristocrates? Les partisans de la noblesse, les optimates du monde romain? Ou les meilleurs au sens moral, les boni viri dont rêvait Cicéron?

On le voit, traduire ici, c'est interpréter, prendre parti et déjà commenter. En effet adopter une traduction unique et mécanique pour chacun de ces termes nous paraît une solution de facilité qui n'aurait aucun sens, dans la mesure où la polysémie de la "langue source " et de la "langue cible" ne sont pas superposables. Et il nous semble nécessaire d'éviter tout esprit de système, si rassurant qu'il puisse être.

Nous nous sommes donc décidée au cas par cas, en tâtonnant. À nos yeux, il est très important de garder en mémoire que le mot " texte " signifie étymologiquement " tissu ». Chaque texte, chaque tissu, a ses lois propres, et un vocable peut changer de signification selon le 
contexte dans lequel il apparait... Dans l'idéal, il faudrait rendre "ce que les mots ne disent pas, mais ce qu'ils font ${ }^{(27) ~ » ~: ~ l e u r s ~ r e t o u r s, ~ l e u r s ~}$ altérations et leurs allitérations, leurs assonances, leurs jeux d'échos et d'opposition...

Dans l'idéal... nous sommes bien consciente que nous n'atteindrons jamais cet idéal, et qu'il n'y aura jamais «la » bonne traduction. Aucune d'elles n'est définitive. Chacune en dit sûrement plus long sur son auteur et sur l'époque à laquelle elle a été produite que sur l'objet dont elle prétend rendre compte. D'où l'intérêt historique des retraductions successives et la nécessité de les étudier en tant que telles, dans une perspective proprement épistémologique.

"Étrangère, désespérément étrangère ", écrit Moses Finley à la suite de Jones, à propos de la tragédie grecque(28). Après avoir consacré des années à l'enseignement et à la traduction des langues anciennes, nous sommes convaincue que notre sensibilité de Modernes ne sera jamais celle d'un Athénien du ve siècle ou d'un Romain de l'époque d'Auguste. L'Antiquité nous échappera toujours, et il serait non seulement illusoire, mais très dangereux moralement de chercher en elle une autorité, un miroir, des " racines".

Cette restriction fondamentale étant posée, il ne faut pas renoncer à lire, à traduire et à retraduire les textes anciens. Bien au contraire. Comme l'écrit Moses I. Finley, "plus notre écoute du passé est précise, plus nous sommes conscients de son caractère passé ou même de sa quasi-inaccessibilité, plus le dialogue prend de sens. Il n'y aura jamais

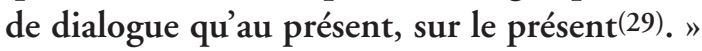

(27) Meschonnic : 1999.

(28) Finley : 1999.

(29) Op. cit., p. 12. 
Chaque traducteur tente à sa manière de dialoguer avec le texte. Il essaie de l'apprivoiser, de lui donner une « auberge " pour reprendre la belle image d'Antoine Berman(30), ou, pour citer Plutarque lui-même, de lui " offrir l'hospitalité ", de lui permettre "d'habiter et de vivre avec nous(31). " Savoir que cette " auberge " est fragile et n'offre qu'un abri provisoire, ne fait-il pas de nous des "hôtes" (et l'on sait combien ce mot est riche et complexe dans le monde antique !) moins autoritaires et donc plus respectueux?

Anne-Marie Ozanam est professeur de lettres supérieures au lycée Henri IV de Paris. Elle y enseigne le latin et le grec ancien. Elle a publié plusieurs traductions, commentées ou non, de textes latins ou grecs, notamment La Guerre, Trois tacticiens grecs : Énée, Asclépiodote, Onasandre (traduction avec Pascal Charvet de textes présentés par Olivier Battistini) Paris, 1994 ; La Magie, voix secrètes de l'Antiquité (traduction et présentation des textes avec Pascal Charvet) Paris, 1994 ; Tacite, Agricola et Germania (introduction, notes et traduction de l'Agricola ; introduction et notes de la Germania) Paris, 1997 ; César, Guerre des Gaules, I-II (traduction), Paris, 1997 ; Alciphron, Lettres de pêcheurs, de paysans, de parasites et d'hétaïres (introduction, traduction et notes), Paris 1999 ; Plutarque, Vies parallèles (traduction), Paris, 2001. 


\section{Bibliographie}

Bélis, Annie, Les Musiciens dans l'Antiquité, Paris, 1999.

Berman, Antoine, L'Épreuve de l'étranger, Paris, 1984.

Berman, Antoine, La traduction et la lettre ou l'auberge du lointain, rééd. Paris, 1999.

Bernand, André, Sorciers grecs, Paris, 1991.

Canfora, Luciano, La Germania di Tacito da Engels al Nazismo, Naples, 1979.

Carré, Irénée, Les Pédagogues de Port-Royal, Paris, 1887.

Charvet, Pascal et Ozanam, Anne-Marie, La Magie, Voix secrètes de l'Antiquité, Paris, 1994.

Chervel, André, Les Auteurs français, latins et grecs au programme de l'enseignement secondaire de 1800 à nos jours, Paris, 1986.

Dodds Eric-Robertson, Les Grecs et l'irrationnel, Berkeley 1951 (trad. Paris, 1981).

Dubois, Claude-Gilbert, Celtes et Gaulois au XVIe siècle, Paris, 1972.

Dumézil, Georges, Les Dieux des Germains, 2e éd., Paris, 1959.

Ginzburg, Carlo, Le Sabbat des sorcières, Turin, 1989, trad. Paris, 1992.

Grimal, Pierre, Tacite, Paris, 1990.

Hartog, François, Le Miroir d'Hérodote, Paris, 1991.

Moses, I Finley, On a perdu la guerre de Troie, Paris, 1989.

Lloyd, Geoffrey E. R., Pour en finir avec les mentalités, Cambridge, 1990 (trad. Paris, 1993).

Meschonnic, Henri, Poétique du traduire, 1999.

Michel, Alain, Tacite et le destin de l'Empire, Paris, 1966. 
Nouilhan, Michèle, Pailler, Jean-Marie et Payen, Pascal, Plutarque, Questions romaines et questions grecques, Paris, 1999.

Ozanam, Anne-Marie, Tacite, Agricola et Germania, 1997.

Ozanam, Anne-Marie, Alciphron, Lettres de pêcheurs, de paysans, de parasites et d'hétaïres, Paris, 1999.

Ozanam, Anne-Marie, Plutarque, Vies parallèles, Paris, 2001.

Payen, Pascal, Article "Amyot " dans le dictionnaire accompagnant Plutarque, Vies parallèles, Paris, 2001.

Perret, Jacques, Tacite, Germania, Paris, 1949.

Ridé, Jacques, "Une réception tendancieuse, la Germanie de Tacite et le moralisme patriotique des humanistes allemands ", Actes du colloque " Histoire et historiographie ", Paris, 1980.

Schaeffner, André, Origine des instruments de musique, Paris, 1936, rééd. 1968.

Vidal-Naquet, Pierre, Le Chasseur noir, Paris, 1981.

Volpilhac-Auger, Catherine, Tacite en France de Montesquieu à Chateaubriand, Oxford, 1993. 